\title{
Non operative conservative management of blunt trauma to the spleen in Children: A Single Tertiary Hospital Experience
}

\author{
Mabrouk Akl ${ }^{1}$, Ayman Albaghdady ${ }^{2}$, Ahmed Elshazly ${ }^{3}$, Hala Fouad C, Hany Embaby ${ }^{4}$, Mary KeriakosN, \\ Mahmoud Elshahawy ${ }^{4}$, Diaa EldeebN, Mohamed ElshafeyN, Abdelrahman Elsabaa ${ }^{4}$. \\ ${ }^{1}$ Alazhar University (Pediatric surgery department), ${ }^{2}$ Ain Shams University, ${ }^{3}$ Banha University, C: Cairo University, ${ }^{4}$ \\ Nasser institute for research and treatment
}

\begin{abstract}
Background: Splenic injury is the leading cause of major bleeding in the patients of blunt abdominal trauma. In earlier medical practice, the blunt splenic injury was managed surgically in most cases, but the increased understanding of the splenic function in the immunological process and the identification of post-splenectomy complications have led physicians to prefer the non-operative management .

Material and methods: A retrospective study of the patients with blunt trauma to the spleen in the pediatric age group between the year 2015 and 2018. The study was conducted in (Nasser Institute Hospital for research and treatment). Management of the patients was done using hypovolemic resuscitation and serial follow up. Abdominal ultrasound, complete blood picture and Abdominal CT were done. Data was tabled and analyzed.

Results: A total of 123 blunt splenic trauma patients were identified. Traffic-related accident and falling from height were the main mechanisms of injury. Splenic contusion and hematoma were the most frequent finding on initial computerized tomography (CT) scans, followed by shattered spleen, blush, and devascularization. Non-operative management failed in 6 patients who underwent splenectomy. (95.12\%) of the patients managed successfully using hypovolemic resuscitation without need for surgical interference.

Discussion: Non-operative management is considered for patients with low-grade splenic injuries, unless operated upon for other associated injuries. In fact, higher-grade injuries could also be managed nonoperatively. Splenic angioembolization of bleeding vessels increased the success rate of non-operative management in hemodynamically stable patients. In our study, although we didn`t use splenic angioembolization we were able to reach high rates of success. In comparison with the operative management of blunt splenic trauma, the non-operative management has the added benefit of preserving the splenic functions.

Conclusions: Most blunt splenic trauma patients were successfully treated non-operatively, with a low failure rate. The severity of injury and presence of associated lesions should be carefully considered in developing the management plan.
\end{abstract}

Key Words: Spleen, Pediatric, Injury, Non-operative management, NOM.

\section{Introduction}

Blunt trauma is one of the most common causes of morbidity and mortality in children, In spite of the great prevention efforts, blunt abdominal trauma (BAT) still remains a leading cause of morbidity and mortality especially in children. The mode of trauma is variable but the most common cause is traffic accident, which represents about $75-80 \%$. Other causes include falling from a height, bicycle handlebar injuries, injuries in contact sports or child abuse. The organs most commonly injured are the spleen and the liver followed by the kidney, the pancreas, the urinary bladder and hollow viscera ${ }^{(1)}$. As the spleen is one of the most commonly injured organ in blunt abdominal trauma patients, its management was a point of controversy.

The risk of laparotomy, the discovery of the immunological role of the spleen and the recognition of the risk of the overwhelming postsplenectomy infection (OPSI), all these factors, made the nonoperative management of blunt splenic injuries is the treatment of choice in hemodynamically stable patients ${ }^{(2)}$. The Advantages of the nonoperative management include a decreased lifetime risk of developing overwhelming postsplenectomy infection, a shorter hospital stay, fewer blood transfusions, and a 
decreased overall mortality and morbidity. Despite of these advantages, adult trauma surgeons are still less likely than pediatric surgeons to proceed into a nonoperative management plan when presented with the same clinical scenario of pediatric solid organ injuries ${ }^{(3)}$.

\section{Material and methods}

The records of patients who were treated in the Pediatric Surgery Department of Nasser Institute Hospital for research and treatment from Jan 2015 to Jan 2018 for abdominal blunt trauma were retrospectively analyzed. Type of injury, injured organ and mode of treatment were recorded. Along with demographic data of patients, non-operative treatment failure and mortality were also recorded.

In our Department, blunt abdominal trauma patients who were hemodynamically stable and without any signs of peritonitis underwent conservative non-operative management for their abdominal injuries using

hypovolemic resuscitation and serial follow up (abdominal ultrasound, complete blood picture and abdominal CT). These patients were followed up closely with physical examination by the same physician. Informed consent was obtained from all patients. Patients were operated if resistant deterioration of hemodynamic stability developed.

Our management plan depended on the hypovolemic resuscitation technique by giving the patient $90 \%$ of its intravenous fluids need. the key point was to keep the patient's blood pressure at the lower edge of its normal range to allow the hemostatic process to function more efficiently. In figure (1), the guidelines used for management are described.

Figure (1): ATOMAC algorithm ${ }^{(4)}$

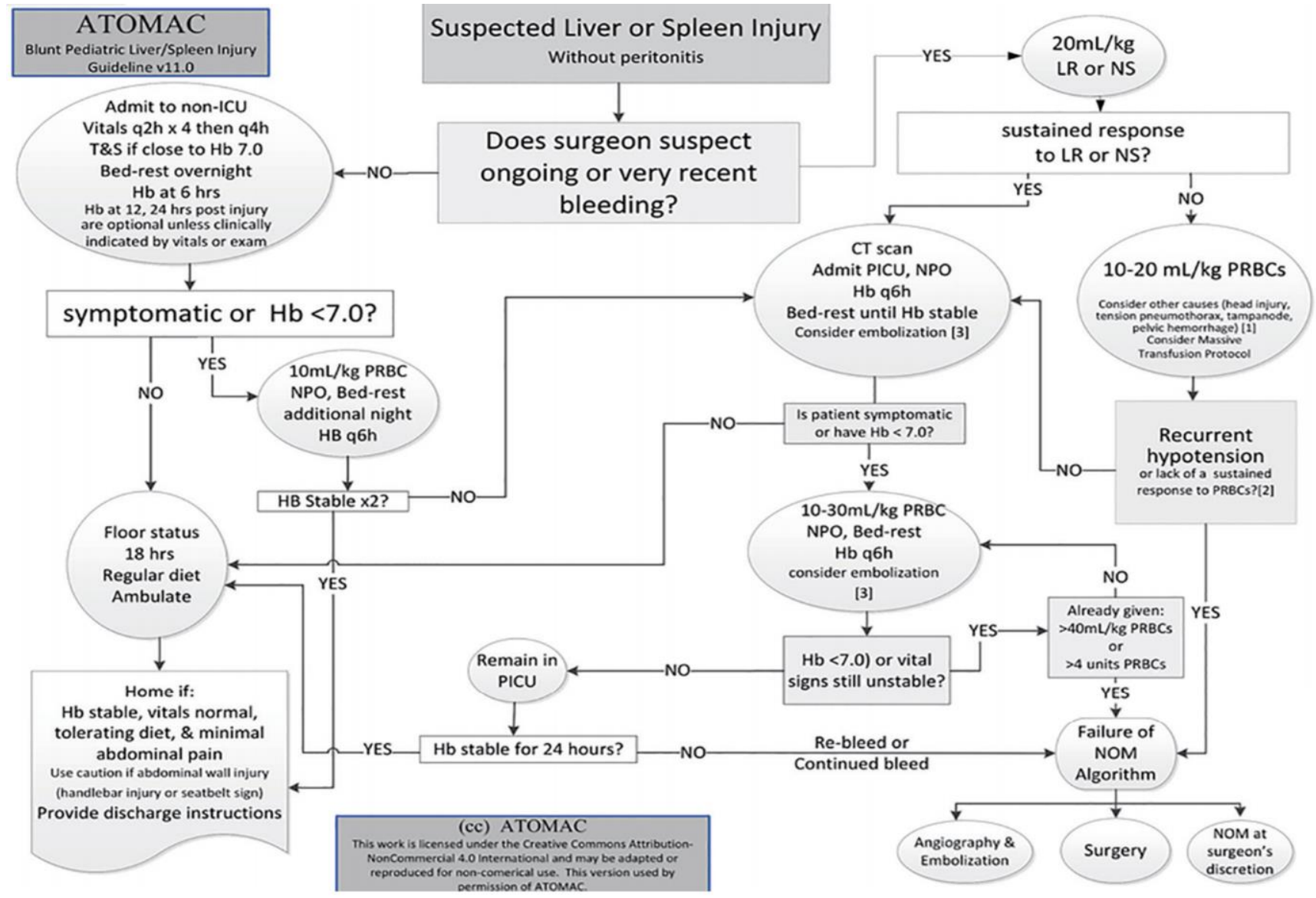


In our study, a total of 123 blunt splenic trauma pediatric patients were included. Patients with associated solid organ injuries were excluded from our study. All of these Patients were subjected to initial resuscitation followed by focused assessment with sonography for trauma (FAST) and once the patient was stable, CT abdomen and pelvis with IV contrast was done to determine the grade of the splenic injury according to the American Association for the Surgery of Trauma Organ Injury Scale (table 1$)^{(5)}$. The patient was then admitted to the ward or the pediatic ICU and managed according to the guidlines described in figure (1).

Table (1): Spleen OIS ${ }^{(5)}$. Advance one grade for multiple injuries up to grade III

\begin{tabular}{lll}
\hline Grade & Injury type & Description of injury \\
\hline I & Hematoma & $\begin{array}{l}\text { Subcapsular, }<10 \% \text { surface area. } \\
\text { Capsular tear, }<1 \mathrm{~cm} \text { parenchymal depth. }\end{array}$ \\
\hline II & Hematoma & $\begin{array}{l}\text { Subcapsular, } 10-50 \% \text { surface area. } \\
\text { Intraparenchymal, }<5 \mathrm{~cm} \text { in diameter. } \\
\end{array}$ \\
& Laceration & $\begin{array}{l}\text { Capsular tear, } 1-3 \mathrm{~cm} \text { parenchymal depth that does not involve a } \\
\text { trabecular vessel. }\end{array}$ \\
\hline III & Hematoma & $\begin{array}{l}\text { Subcapsular, }>50 \% \text { surface area or expanding; ruptured } \\
\text { subcapsular or parenchymal. }\end{array}$ \\
& Laceration & 3 cm parenchymal depth or involving trabecular vessels. \\
\hline IV & Laceration & $\begin{array}{l}\text { Laceration involving segmental or hilar vessels producing major } \\
\text { devascularization. }\end{array}$ \\
\hline V & Laceration & Completely shattered spleen. \\
& Vascular & Hilar vascular injury which devascularizes spleen. \\
\hline
\end{tabular}

\section{Statistical analysis}

Statistical Packages for the Social Sciences (SPSS) version 20.0 for Windows program was used for the analysis of data. Descriptive statistics were presented as mean and standard deviation. Categorical data were analyzed by chi-square test. Significance level was accepted as $\mathrm{p}<0.05$.

\section{Results}

A total of 123 blunt splenic trauma patients were included in our study. Their ages ranged from 2 to 12 years old with mean age of 6.43 years old. $67.5 \%$ were males and $32.5 \%$ were females. The mode of injury was described in table (2).

Table (2): The mode of injury and its rate

\begin{tabular}{l|l|l}
\hline The mode of injury & Number of patients & Percentage \\
\hline Traffic-related accident & 73 & $59.35 \%$ \\
\hline Falling from height & 36 & $29.27 \%$ \\
\hline Animal related injury & 4 & $3.25 \%$ \\
\hline bicycle handlebar injuries & 4 & $3.25 \%$ \\
\hline contact sports & 6 & $4.88 \%$ \\
\hline child abuse & 0 & $0 \%$ \\
\hline
\end{tabular}


Traffic-related accident and falling from height were the main mechanisms of injury with combined total percentage of 88.62 (TRA was $59.34 \%$ and FFH is 29.26\%). Other modes of injury included, animal related injury, bicycle handlebar injuries and injuries in contact sports or child abuse with combined percentage of 11.38 .

Table (3): The grade of injury
Splenic contusion and hematoma were the most frequent finding on initial computerized tomography (CT) scans, followed by shattered spleen, blush, and devascularization. Grading of the splenic injuries was done using the American Association for the Surgery of Trauma Organ Injury Scale and was described in table 3

\begin{tabular}{|l|l|l|}
\hline The grade of injury & Number of patients & Percentage \\
\hline I & 39 & $31.71 \%$ \\
\hline II & 35 & $28.46 \%$ \\
\hline III & 30 & $24.39 \%$ \\
\hline IV & 15 & $12.19 \%$ \\
\hline V & 4 & $3.25 \%$ \\
\hline
\end{tabular}

The majority of the patients suffered from an injury grade I-III with percentage of 84.56, while grade IV injury represented only a percentage of 12.19 and grade $\mathrm{V}$ injury represented only a percentage of 3.25.

Non-operative management was successful in about 117 patients with percentage of 95.12, while 6 patient showed hemodynamic instability and failure of NOM and underwent splenectomy with percentage of 4.88. Two of these 6 patients required immediate laparotomy and splenectomy, while the other 4 was operated the second day after admission due to hemodynamic instability inspite of resusscitative measures and packed RBCs transfussion. These 4 patients also underwent laparotomy and splenectomy.

\section{Discussion}

Non-operative Management is the "gold standard" for pediatric patients with blunt abdominal trauma that are clinically stable. As children have smaller blood vessels and more efficient vasoconstrictive response. Splenic capsule and vessel injury usually stop bleeding spontaneously regardless of injury type and grade. Consequently, most patients will respond well to non-operative management. Our personal experience support the principle of conservative non-operative management in all haemodynamically stable patients, with overall efficacy rate more than $91 \%$, as was reported in modern literature ${ }^{(6,7)}$.

The grade of the splenic injury is an important factor affecting the success of nonoperative management. the higher the grade of splenic injury according to the American Association for the Surgery of Trauma Organ Injury Scale (OIS) the higher the risk of failure of $\mathrm{NOM}^{(5)}$.

The Research Consortium of New England Centers for Trauma (ReCONECT) examined in New England trauma centers the outcomes of only grade IV and V blunt splenic injuries in 14 trauma centers from 2001 to $2008^{(6)}$. They studied 388 patients, with approximately about 4 grade IV patients for every one-grade $\mathrm{V}$ patient. Of grade IV patients, about $38 \%$ underwent immediate laparotomy and a third of those who managed 
using NOM eventually required laparotomy. Of grade $\mathrm{V}$ patients, about $60 \%$ underwent immediate operation and another $25 \%$ failed NOM.

Quantity of hemoperitoneum can also be assessed using Focused assessment with sonography for trauma (FAST) and has been reported in several studies to be associated with failure of $\mathrm{NOM}^{(8,9,10)}$. Moderate to large volumes of hemoperitoneum have been reported in 59-64\% of patients with splenic injury with failure rates of $10-12 \%$. However the quantity of hemoperitoneum alone was not associated with a statistically significant increased risk of failure of $\mathrm{NOM}^{(5)}$.

Splenic artery angiography and embolization has been increasingly considered as a supportive measure to NOM. In the higher grades of blunt splenic injury, the advantage of splenic artery embolization seems to be obvious. A retrospective study from 2012 reported on the selective use of angioembolization in hemodynamically stable adult patients selected for NOM ${ }^{(11)}$. Of the 539 patients, about 435 underwent observation alone $(81 \%)$, while $104(19 \%)$ subjected to angioembolization. Failure rates for grades IIII did not differ whether or not angioembolization was used. On the other hand, in grade IV and V injuries, failure of NOM was significantly decreased with the addition of angiography and embolization (23 vs. $3 \%$, and 63 vs. $9 \%$, respectively) ${ }^{(11)}$.

In 2017 WSES classification for splenic injuries, a new classification and guidelines, was announced. The WSES paper suggested to group splenic injury into 3 groups minor, moderate, and severe. Previously lowgrade AAST lesions (grades I to III) are considered as minor or moderate and treated with NOM. However, hemodynamically stable patients with high grade lesions could be also successfully treated non-operatively, especially with the advancements in bleeding management. On the other hand, minor lesions with hemodynamic instability usually must be treated with laparotomy ${ }^{(12)}$.

The new WSES classification divides spleen injuries into three classes, depending on both the anatomic AAST-OIS classification and the hemodynamic status. Minor (WSES class I), moderate (WSES classes II and III) and severe (WSES class IV) ${ }^{(12)}$.

Minor spleen injuries is WSES class I which includes hemodynamically stable AAST-OIS grade I-II blunt and penetrating lesions. Moderate spleen injuries is WSES class II and III. WSES class II, which includes hemodynamically stable AAST-OIS grade III blunt and penetrating lesions and WSES class III which includes hemodynamically stable AAST-OIS grade IV-V blunt and penetrating lesions. Severe spleen injuries is WSES class IV which includes hemodynamically unstable AAST-OIS grade I-V blunt and penetrating lesions ${ }^{(12)}$. Based on that classification, WSES suggests a new management algorithms as in table (4) and figure (2).

\section{conclusion}

The results of this study donates that conservative treatment is the gold standard for all stable patients with blunt splenic trauma regardless of the grade of the organ injury. The choice of non-operative treatment should be based mainly on physiological response of the child, rather than the injury grade on CT scan. Assessment of haemodynamic stability is the most important initial concern in the evaluation of a patient with blunt abdominal trauma. WSES Spleen Trauma Classification depending on both the anatomic AAST-OIS classification and the hemodynamic status and can be used to improve the outcome of NOM. 
Non operative conservative management of blunt trauma to the spleen in Children...

\begin{tabular}{|c|c|c|c|c|c|c|}
\hline & WSES class & Mechanism of injury & AAST & Hemodynamic Status & CT scan & First-line treatment in pediatric \\
\hline Minor & WSES I & Blunt/penetrating & I-II & Stable & \multirow{3}{*}{$\begin{array}{l}\text { Yes + local } \\
\text { exploration in } \\
\text { case of stab } \\
\text { wound }\end{array}$} & $\mathrm{NOM}+$ serial \\
\hline \multirow[t]{2}{*}{ Moderate } & WSES II & Blunt/penetrating & III & Stable & & clinical/laboratory/radiological evaluation. \\
\hline & WSES III & Blunt/penetrating & IV-V & Stable & & Consider angiography/Angioembolization \\
\hline Severe & WSES IV & Blunt/penetrating & $\mathrm{I}-\mathrm{V}$ & Unstable & No & $\mathrm{OM}$ \\
\hline
\end{tabular}

\section{PEDIATRIC PATIENTS}

\section{Spleen Trauma}

In the E.D.: FAST-E, Thoracic and Pelvic X-ray,

Hemodynamically Stable

US \pm Contrast Enhanced CT-Scan

+ Local Exploration in SW \#

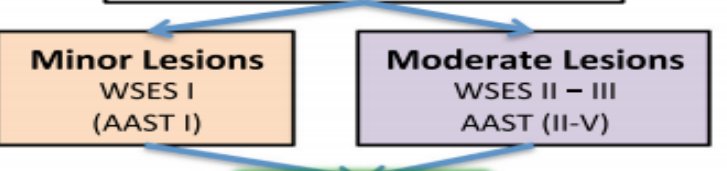

NOM *
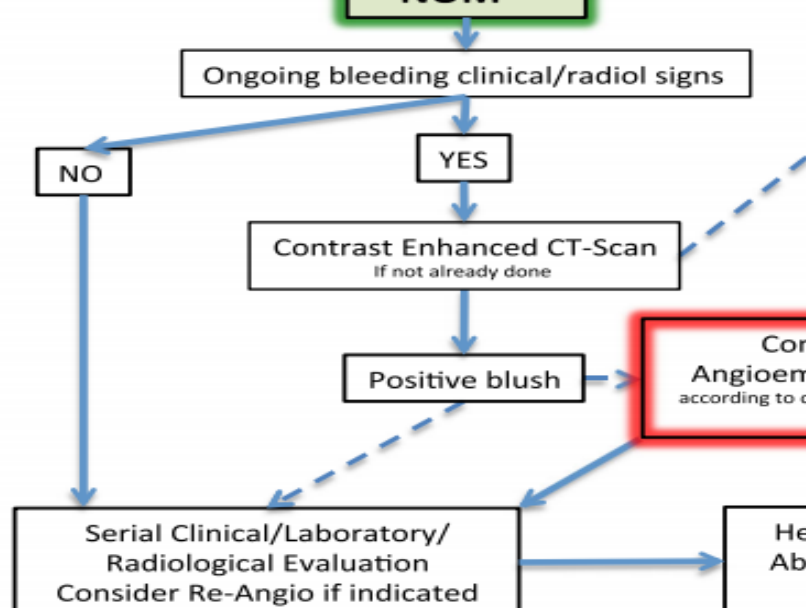

Consider Re-Angio if indicated
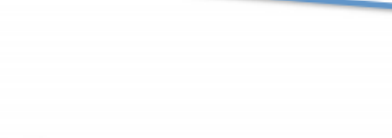

Hemodynamically Unstable @

$$
\text { :-POSitive E-FAST'i }
$$

Severe Lesions

WSES IV
(AAST I-V)

(AAST I-V)

Laparotomy

\pm Partial/Total

Splenectomy

figure (2): WSES Spleen Trauma Management Algorithm for Pediatrics Patients ${ }^{(12)}$. 


\section{Refrences}

1. Djordjevic I, Slavkovic A, Marjanovic Z, Zivanovic D (2015): Blunt trauma in paediatric patients - experience from a small centre. West Indian Med J. ,64 (2): 126-130.

2. Skattum J, Naess P A, Gaarder C (2012): Non-operative management and immune function after splenic injury. British journal of surgery, 99 (1): 59-65.

3. Singer G, Rieder S, Eberl R, Wegmann H, Hoellwarth M E (2013): Comparison of two treatment eras and sonographic long-term outcome of blunt splenic injuries in children. Eur J Pediat.r, 172: 1187-1190.

4. Minaya-Bravoa A M, Aguado-Lopez $\mathbf{H}$ (2017): The Challenge of Blunt Abdominal Trauma in Children: Report of a Case and Review of Management. J Med Cases, 8 (11): 340-346.

5. Watson G A, Hoffman M K, Peitzman A B (2015): Nonoperative management of blunt splenic injury: what is new? Eur J Trauma Emerg Surg., 41: 219-228.

6. Velmahos G C, Zacharias N, Emhoff T A et al. (2010): Management of the Most Severely Injured Spleen: A Multicenter Study of the Research Consortium of New England Centers for Trauma (ReCONECT). Arch Surg., 145 (5): 456460.
7. Harbrecht B G, Ko S H, Watson G A, Forsythe R M, Rosengart M R, Peitzman A B (2007): Angiography for blunt splenic trauma does not improve success rate of non-operative management. J Trauma, 63: 44-49.

8. Peitzman A B, Heil B, Rivera L, Federle M B et al.(2000): Blunt splenic injury in adults: multi-institutional study of the Eastern Association for the Surgery of Trauma. J Trauma-Inj Infect Crit Care, 49: 177-189.

9. Bee $T \mathrm{~K}$, Croce M A, Miller P R, Pritchard F E, Fabian T (2001): Failures of splenic nonoperative management: is the glass half empty or half full? J Trauma Acute Care Surg., 50: 230-236.

10. Haan J M, Bochicchio G V, Kramer N, Scalea T M (2005): Nonoperative management of blunt splenic injury: a 5year experience. J Trauma-Inj Infect Crit Care, 58: 492-498.

11. Bhullar I S, Frykberg E R, Siragusa D et al. (2012): Selective angiographic embolization of blunt splenic traumatic injuries in adults decreases failure rate of nonoperative management. J Trauma Acute Care Surg., 72: 1127-1134.

12. Coccolini F, Montori G, Catena F et al. (2017): Splenic trauma: WSES classification and guidelines for adult and pediatric patients. World J Emerg Surg., 18: $12-40$ 